\title{
Identification of main effect and epistatic quantitative trait loci for morphological and yield-related traits in peanut (Arachis hypogaea L.)
}

\author{
Yogendra Khedikar • Manish K. Pandey • V. Sujay • Sube Singh • Spurthi N. Nayak • \\ Henry W. Klein-Gebbinck • Cholin Sarvamangala • Ganapati Mukri • Vanika Garg • \\ Hari D. Upadhyaya • H. L. Nadaf • M. V. C. Gowda • Rajeev K. Varshney • \\ Ramesh S. Bhat
}

Received: 4 May 2017 / Accepted: 4 December 2017

(C) Springer Science+Business Media B.V., part of Springer Nature 2017

\begin{abstract}
An effort was made in the present study to identify the main effect and epistatic quantitative trait locus (QTL) for the morphological and yield-related traits in peanut. A recombinant inbred line (RIL) population derived from TAG $24 \times$ GPBD 4 was phenotyped in seven environments at two locations. QTL analysis with available genetic map identified 62 main-effect
\end{abstract}

Yogendra Khedikar and Manish K. Pandey contributed equally to this work.

Electronic supplementary material The online version of this article (https://doi.org/10.1007/s11032-017-0764-z) contains supplementary material, which is available to authorized users.

Y. Khedikar · V. Sujay · C. Sarvamangala · G. Mukri • M. V. C. Gowda

Department of Genetics and Plant Breeding, University of Agricultural Sciences, Dharwad, India

e-mail: r.k.varshney@cgiar.org

Y. Khedikar · M. K. Pandey · V. Sujay · S. Singh •

S. N. Nayak · C. Sarvamangala • V. Garg ·

H. D. Upadhyaya · R. K. Varshney

International Crops Research Institute for the Semi-Arid Tropics (ICRISAT), Hyderabad, India

S. N. Nayak $\cdot$ H. L. Nadaf $\cdot$ R. S. Bhat $(\square)$

Department of Biotechnology, University of Agricultural

Sciences, Dharwad, India

e-mail: bhatrs@uasd.in

H. W. Klein-Gebbinck

Agriculture and Agri-Food Canada, Beaverlodge Research Farm, Beaverlodge, Alberta, Canada
QTLs (M-QTLs) for ten morphological and yieldrelated traits with the phenotypic variance explained (PVE) of 3.84-15.06\%. Six major QTLs (PVE > 10\%) were detected for PLHT, PPP, YPP, and SLNG. Stable M-QTLs appearing in at least two environments were detected for PLHT, LLN, YPP, YKGH, and HSW. Five M-QTLs governed two traits each, and 16 genomic regions showed co-localization of two to four M-QTLs. Intriguingly, a major QTL reported to be linked to rust resistance showed pleiotropic effect for yield-attributing traits like YPP (15.06\%, PVE) and SLNG (13.40\%, PVE). Of the 24 epistatic interactions identified across the traits, five interactions involved six M-QTLs. Three interactions were additive $\times$ additive and remaining two involved QTL $\times$ environment $(\mathrm{QE})$ interactions. Only one major M-QTL governing PLHT showed epistatic interaction. Overall, this study identified the major MQTLs for the important productivity traits and also described the lack of epistatic interactions for majority of them so that they can be conveniently employed in peanut breeding.

Keywords Peanut - QTL analysis · Epistatic interaction · Agro-morphological traits · Yield

\section{Introduction}

Peanut (Arachis hypogaea L.) is a legume and oilseed crop with high protein content. Globally, it is grown in 
an area of 25.7 million ha with the production of 42.3 million tons (FAOSTAT 2016). Enhancing the overall productivity by improving the traits like resistance to diseases, tolerance to drought, enhanced oil content, and improved quality traits is the main objective in peanut breeding. However, many of these traits are genetically complex, and achieving significant gains therefore requires the applications of genomics-assisted breeding (GAB) (Varshney et al. 2013).

There has been a tremendous advancement over the last decade in understanding the peanut genome. The genomes of the diploid progenitor species of cultivated peanut have been sequenced (Bertioli et al. 2016; Chen et al. 2016b). Recently, the chloroplast genomes of seven species have also been sequenced (Yin et al. 2017) to understand the genetic relationships of the wild species with the cultivated peanut. A molecular phylogeny based on the complete chloroplast genome sequences provided the best resolution of the seven Arachis species. A large number of different types of markers have been developed (Zhao et al. 2017), and a high-throughput genotyping assay with $58 \mathrm{~K}$ informative single nucleotide polymorphism (SNP) markers was developed (Pandey et al. 2017a). With these markers, the genetic maps of varying saturation levels have been developed and employed for mapping various traits (Vishwakarma et al. 2017). Genome-wide association studies were also conducted to map the traits like disease resistance, quality, drought tolerance and yield components (Pandey et al. 2014a) and agronomic traits (Zhang et al. 2017). Further, QTL-Seq has identified the candidate genes for LLS and rust resistance (Pandey et al. 2017b). Also, the RNA-Seq study has revealed the transcripts that are differentially expressed under LLS infection (Han et al. 2017).

The markers identified for foliar disease resistance were validated (Sujay et al. 2012; Yeri et al. 2014; Sukruth et al. 2015) and employed for molecular breeding (Varshney et al. 2014; Yeri and Bhat 2016). Markerassisted breeding has also been successful in enhancing the oleic acid content and resistance to nematode. However, the molecular breeding for other traits in peanut demands a thorough detection and analysis of QTL and markers. Identification of QTL with main effects (or individual effects), epistatic effects, and $\mathrm{G} \times$ $\mathrm{E}$ interactions is very useful in genomics (Carlborg and Haley 2004). In peanut, many researchers have reported additive, non-additive (including epistatic genetic) effects for traits like pod yield, number of pods and seeds per plant, hundred seed weight, pod length, and shelling outturn based on the genetic analysis (Layrisse et al. 1980; Dwivedi et al. 1989; Upadhyaya and Nigam 1998). However, identification of M-QTLs, E-QTLs, and G $\times$ $\mathrm{E}$ interactions for the agro-morphological and productivity traits will be useful for molecular breeding in peanut.

Availability of an appropriate mapping population with a large number of recombinants, phenotyping over multi-environments, and multi-seasons would allow precise detection of reliable (consistent) QTLs for the target traits. Therefore, the present study employed the RIL mapping population derived from TAG $24 \times$ GPBD 4 for identifying the M-QTLs, epistatic-QTLs, and QTL $\times$ environment $(\mathrm{QE})$ interactions for the important agromorphological and yield traits in peanut.

\section{Materials and methods}

\section{Plant materials}

This study used a mapping population consisting of 268 RILs of TAG $24 \times$ GPBD 4 developed at the University of Agricultural Sciences (UAS), Dharwad, India. GPBD 4 is a very popular and high-yielding Spanish bunch cultivar with resistance to foliar fungal diseases, early maturity, desirable pod and kernel features, and high oil content (Gowda et al. 2002). TAG 24 is a Spanish bunch cultivar (Patil et al. 1995) which suffers significant yield loss under severe foliar disease infection.

Phenotyping and statistical analysis of mapping populations

The RIL-4 mapping population of $\mathrm{F}_{9-12}$ generations was phenotyped for three environments namely post-rainy 2007 (R4EI), rainy 2007 (R4EII), and post-rainy 2008 (R4EIII) at ICRISAT, Patancheru, India $\left(17^{\circ} 30^{\prime} 39.8^{\prime \prime} \mathrm{N}\right.$ $78^{\circ} 16^{\prime} 30.8^{\prime \prime E}$ ), and four environments at UASDharwad, India (15²9'30.1"N 7459'00.9"E), viz., rainy 2007 (R4EIV), rainy 2008 (R4EV), rainy 2009 (R4EVI), and post-rainy 2009 (R4EVII). The RILs were planted in augmented plot design with parents as checks in 15 blocks of 20 rows in R4EI; Alpha design with three replications in R4EII and two replications in R4EIII, and randomized block design (RBD) with two replications in R4EIV, R4EV, R4EVI, and R4EVII. Observations on 14 agro-morphological traits, namely, days to flowering (DF), plant height (PLHT), leaf length 
(LLN), leaf width (LWD), pod length (PLN), pod width (PWD), seed length (SLN), number of primary branches (PBR), pods per plant (PPP), yield per plant (YPP), yield in $\mathrm{kg}$ per hectare (YKGH), hundred seed weight (HSW), shelling percentage (SLNG), and days to maturity (DM) were recorded at ICRISAT-Patancheru. The RILs were phenotyped for 12 yield-contributing traits at R4EI and all 14 traits measured in R4EII and R4EIII. A total of eight traits viz., PLHT, LLN, LWD, PBR, PPP, YPP, HSW, and SLNG were phenotyped at R4EIV and R4EV; four traits, PLHT, PBR, LLN, and LWD at R4EVI and five yield-contributing traits, PLHT, PPP, YPP, HSW, and SLNG at R4EVII.

Restricted Maximum Likelihood (REML) analysis was performed using GenStat version 12.0 (GenStat Committee 2010) to estimate the variance components for the data collected at ICRISAT, Patancheru. Analysis of variance (ANOVA) was performed using WindowStat, version 8.5 (IndostatServices, Hyderabad, India, http://members.fortunecity.com/indostat/) for the data collected from each season at Dharwad location. The parameters like genetic variance $\left(\sigma_{\mathrm{g}}^{2}\right)$ and broad sense heritability $\left(h_{\text {b.s. }}^{2}\right)$ were estimated using GenStat version 12.0 and WindowStat version 8.5 for the data collected at Patancheru and Dharwad location, respectively. Broad sense heritability was calculated as $h_{\text {b.s. }}^{2}=\left(\sigma_{\mathrm{G}}^{2} / \sigma_{\mathrm{P}}^{2}\right)$ for each environment where, $\sigma_{\mathrm{G}}^{2}$ represented the genotypic variance, $\sigma^{2}$ is the phenotypic variance $\left(\sigma_{\mathrm{G}}^{2}+\left(\sigma^{2} \mathrm{e}\right.\right.$ or error residual/number of replications). The correlation coefficients ( $r$ ) among the traits were estimated in each season using an R software (R Core Team 2013).

\section{QTL analysis}

The genetic map, carrying 188 mapped markers, constructed previously by Sujay et al. (2012), was used for QTL analysis. Candidate interval selection, putative QTL detection, and QTL effects were estimated from each environment (three from ICRISAT and four from Dharwad) separately. The normalized predicted mean from the REML estimates in Patancheru location and mean phenotypic data across replications in Dharwad location were used to map QTLs by "Composite Interval Mapping (CIM)" approach (Zeng 1994) using WinQTL Cartographer 2.5 (Wang et al. 2007). CIM was carried out using Model 6 with a moving window size of $10 \mathrm{cM}$ and a walking speed of $2 \mathrm{cM}$. The forward-backward stepwise regression method was used to select number of marker cofactors for the background selection. To define QTL region automatically, "Locate QTLs" option was used with a minimum distance of $5 \mathrm{cM}$ between QTLs. The highest peak was considered to locate QTLs if the peak distance between the QTL is less than $5 \mathrm{cM}$. For each trait, a 500 permutation test with a significance level of 0.05 was performed to detect an appropriate LR. Based on significant LR peak for map position under consideration, QTL position, additive effects, favorable allele contribution, and phenotypic variation explained (PVE) were estimated. An estimate of epistatic QTL was studied by mixed linear composite interval mapping (MCIM) using software QTL Network Version 2.0 (Yang et al. 2008). The 2D Genome Scan option was used to map epistatic QTLs. A 1000 permutation was applied to calculate critical $F$ value and the QTL effects were estimated using Monte Carlo Markov chain method with 20,000 Gibs sample size. The genome scan configuration was performed using testing window size of $10 \mathrm{cM}$, walk speed of $1 \mathrm{cM}$, and filtration window size of $10 \mathrm{cM}$.

\section{Results}

Phenotypic variability

REML analysis for the data collected from Patancheru and ANOVA for the data collected from Dharwad showed significant differences among the RILs for majority of the traits except LLN, LWD, YPP, and YKGH in R4EI; DF, SWD, and DM in R4EII; LWD, PBR, SWD, and DM in R4EIII; PBR and LWD in R4EIV; PBR and LWD in R4EV; PBR in R4EVI (Supplementary Table S1). The mean values for all the traits, except PBR in R4EII, were normally distributed (Fig. 1). The phenotypic range of variation was high for all the traits, except for DF in R4EI and R4EIII, PBR in R4EI and R4EII, LWD in R4EII, and PWD and SLN in R4EII and R4EIII. Transgressive segregants were observed for all the traits in all the seven environments at both (Patancheru and Dharwad) the locations. The broadsense heritability estimates $\left(\mathrm{h}_{\text {b.s. }}^{2}\right)$ ranged from low (0.18, YPP in R4EIV), moderate (0.26, HSW in R4EII; 0.30, LWD in R4EII), high to very high (0.91, PPP in R4EV). Briefly, the heritability estimates were observed for DF (0.36-0.85), PLHT (0.43-0.86), LLN (0.38-0.85), LWD (0.30-0.42), PBR (0.38-0.66), PLN (0.54-0.70), PPP (0.32-0.91), PWD (0.38-0.53), SLN 
a
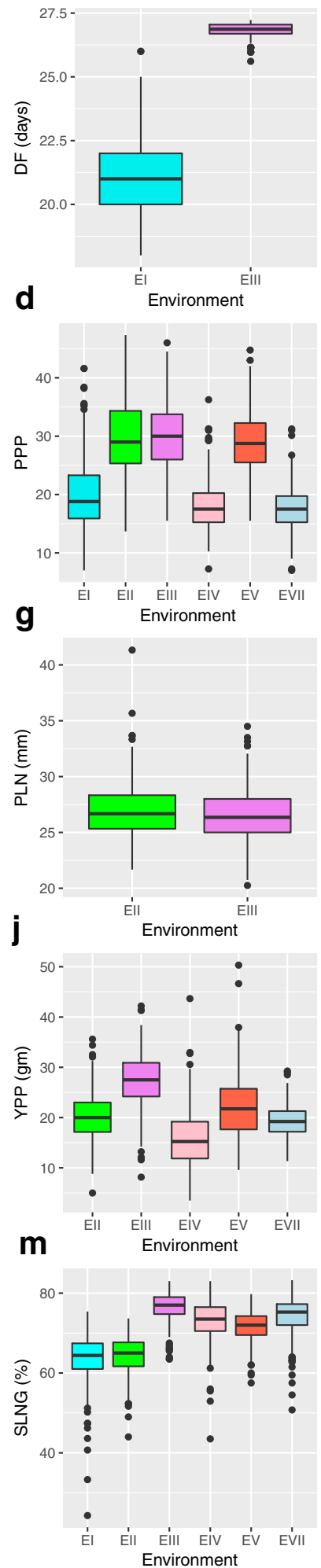

b

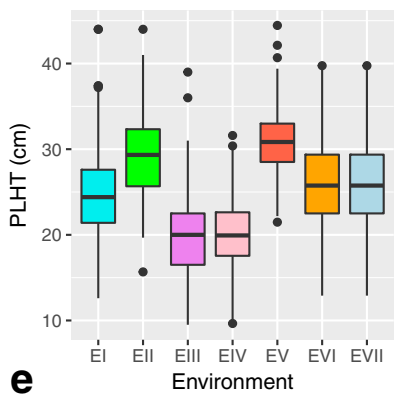

70
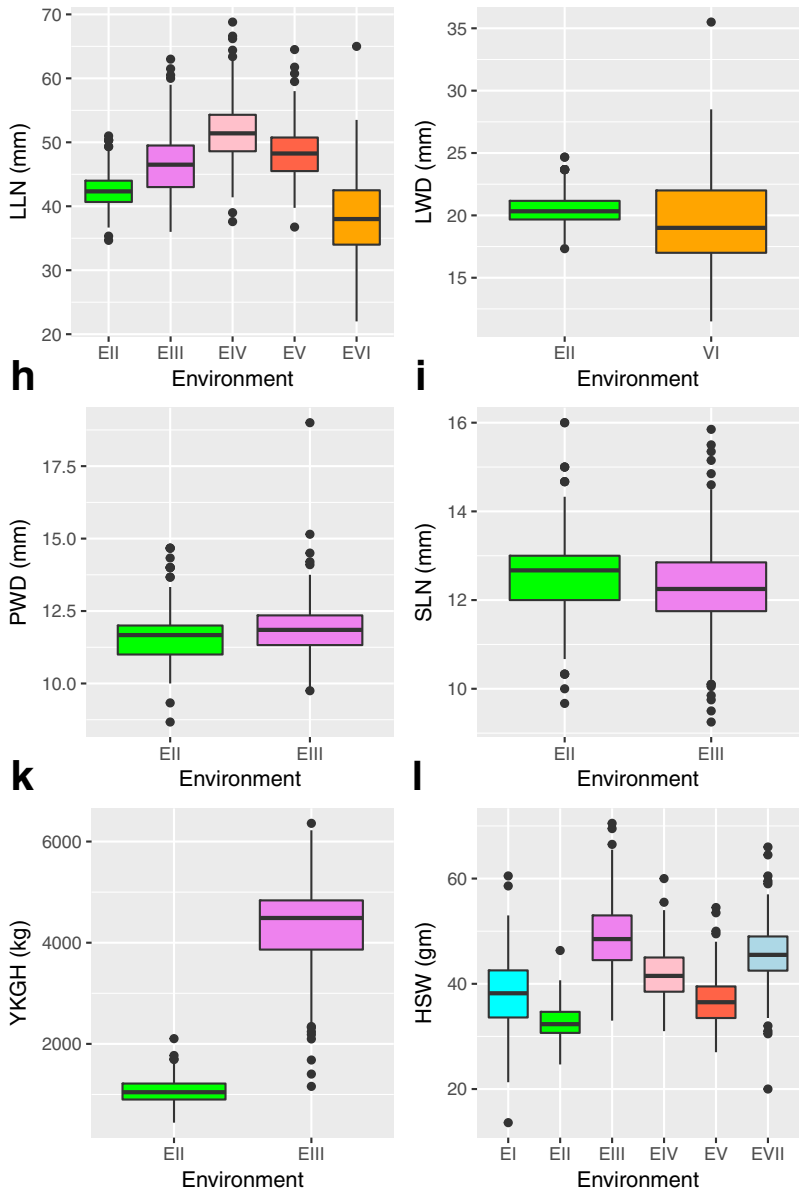
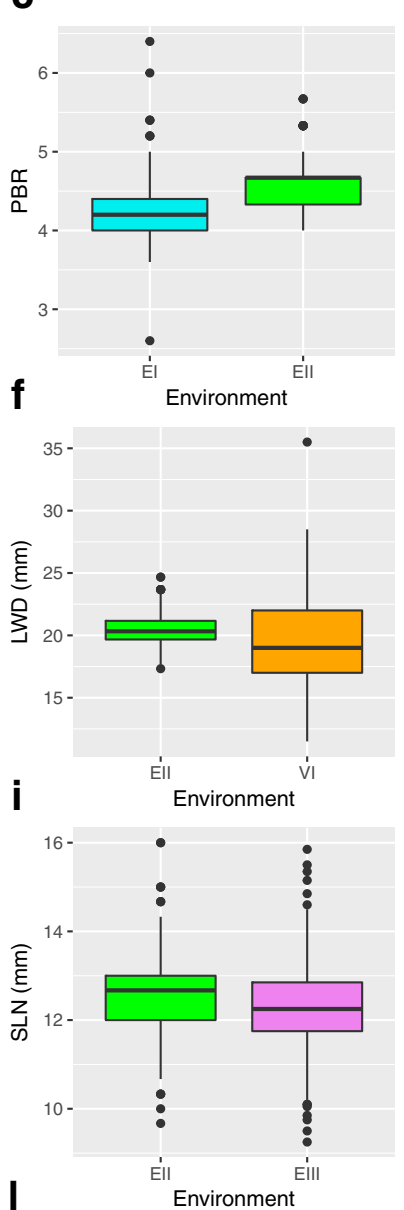

Fig. 1 Boxplot showing the distribution of the means for agro-morphological traits among the RILs over different environments 
(0.66-0.77), YPP (0.18-0.51), YKGH (0.63-0.81), HSW (0.26-0.85), SLNG (0.47-0.82), and DM (0.61).

\section{Phenotypic correlations}

We studied the degree of relationship between the traits in each season by estimating the Pearson's $(r)$ pairwise correlation coefficients (Supplementary Fig. S1). Across all the seasons, PLHT was found positively correlated with LLN, LWD, PWD, PBR, and YPP ( $r=0.13$ to 0.27), however, it was negatively correlated with SLN and SLNG $(r=-0.12$ to -0.15$)$. LLN was positively correlated with pod-attributing traits, namely, PLN, PWD, and SLN ( $r=0.12$ to 0.33 ). Among yieldcontributing traits, PPP showed strong positive correlations with PBR, YPP, and YKGH $(r=0.37$ to 0.78$)$ in Patancheru location and low correlations $(r=0.17$ to 0.22) in Dharwad location. However, PPP was negatively correlated with pod-attributing traits, PLN, PWD, and SLN $(r=-0.18$ to -0.24$)$. Among kernel traits, SLNG was positively correlated with HSW, YPP, and YKGH $(r=0.13$ to 0.36 ), while it was negatively correlated with many agronomic traits, for instance, DF, PLHT $(r=-$ 0.12 to -0.15$)$, SLN, PWD, PLN ( $r=-0.14$ to -0.29$)$, and LLN $(r=-0.14$ to -0.30$)$. HSW showed low to strong positive correlations with SLN, PWD, and PLN $(r=0.26$ to 0.61$)$.

Main effect QTLs (M-QTLs) for agro-morphological traits

The QTL analysis revealed a total of 62 M-QTLs at 39 genomic regions across ten traits, scattered on 15 different LGs. (Table 1; Supplementary Table S2; Fig. 2). M-QTLs could not be detected for LWD, PLN, PWD, and DM. A maximum of $20 \mathrm{M}$-QTLs were detected for PLHT followed by nine for YPP, eight for HSW, and seven each for PPP and SLNG. DF and YKGH had three MQTLs each, while LLN and SLN had two M-QTLs each. PBR was detected with a solitary M-QTL. Five M-QTLs controlled two traits each. For instance, a M-QTL mapped at $65.21 \mathrm{cM}$ at GM1955-GM1007 on $\mathrm{AhV}$ governed PPP and HSW. M-QTL at $0.01 \mathrm{cM}$ in PM377-TC1A01 region on AhVI governed YPP and YKGH. Similarly, a M-QTL at $7.61 \mathrm{cM}$ within TC1A01-S108 contributed for SLN and HSW. PPP and YPP were governed by a single M-QTL $(22.11 \mathrm{cM})$ in the region GM1097-TC7C06 on AhVI.
PPP and YKGH were governed by an M-QTL at $12.51 \mathrm{cM}$ within GM1536-GM2301 on AhXV.

Contribution of M-QTLs in terms of PVE ranged from $3.84-15.06 \%$. Six M-QTLs were considered to be major since the PVE was more than $10 \%$ (Supplementary Fig. S2). The highest PVE of $15.06 \%$ was recorded by a M-QTL at $14.51 \mathrm{cM}$ within IPAHM103-GM1954 on AhXV for YPP. Another MQTL at $20.51 \mathrm{cM}$ with the same marker interval had $13.40 \%$ PVE towards SLNG, which was also governed by single M-QTL at $10.01 \mathrm{cM}$ with GM2009-GM1536 on AhXV with $14.04 \%$ PVE. YPP was governed by a major M-QTL at $15.11 \mathrm{cM}$ within GM1386-GM1162 on AhXIV with PVE of $13.56 \%$. One M-QTL at $61.71 \mathrm{cM}$ within TC3A12-GM1955 on AhV recorded a PVE of $11.57 \%$ for PLHT. Another M-QTL at $60.71 \mathrm{cM}$ within GM1076-Seq5D05 on AhXVIII governed PPP with a PVE of $10.48 \%$. These M-QTLs were detected with high LOD (more than 5.0). Interestingly, the favorable alleles at all the major M-QTLs were contributed by GPBD 4.

The M-QTL at 71.51-73.51 cM within GM633PM179 on AhV with a maximum PVE and LOD of 9.67 and $7.09 \%$, respectively towards PLHT was detected in five different environments. PLHT was also governed by a stable M-QTL at $0.01-1.91 \mathrm{cM}$ within Seq7H06IPAHM176 on AhXII with a maximum PVE and LOD of 7.12 and $5.03 \%$, respectively which appeared across four environments. One M-QTL at $63.21-65.21 \mathrm{cM}$ within GM1955-GM1007 on AhV also controlling PLHT appeared in three environments with a maximum PVE and LOD of 6.18 and $3.49 \%$, respectively. The favorable allele at this stable M-QTL was contributed by GPBD 4. Stable M-QTLs appearing in two environments were detected for LLN, (7.61-12.71 cM, TC1A01S108, AhVI), YPP (56.11-60.11 cM, GM1062GM2638, AhVII), YKGH (0.01 cM, PM377-TC1A01, AhVI), and HSW (70.51-71.51 cM, GM633-PM179, $\mathrm{AhV}$ ). GPBD 4 contributed for the favorable allele at the stable M-QTL for LLN, YPP, and YKGH, while TAG 24 contributed the favorable allele at the stable M-QTL for HSW. However, the major M-QTLs appeared in any one environment.

\section{Co-localization of M-QTLs}

It was interesting to notice that 16 genomic regions consisted of co-localized M-QTLs for various traits (Supplementary Fig. S3). Four genomic regions/ 
Table 1 Main effect QTLs (M-QTLs) detected in composite interval mapping (CIM) analysis for 14 agro-morphological traits in RIL-4 population

\begin{tabular}{lllll}
\hline Trait & Number of M-QTLs & PVE \% & Environment & Source of favorable allele \\
\hline Days to flowering (DF) (days) & 3 & $4.92-6.82$ & R4EI & TAG 24, GPBD 4 \\
Plant height (PLHT) (cm) & 20 & $3.84-11.57$ & R4EI-R4EVII & GPBD 4, TAG 24, \\
Leaf length (LLN) (mm) & 2 & $4.14-8.65$ & R4EIII, R4EIV, R4EV & GPBD 4 \\
Leaf width (LWD) (mm) & - & - & - & - \\
Primary branching (PBR) & 1 & 5.94 & R4EI & GPBD 4 \\
Pods per plant (PPP) (mm) & 7 & $3.91-10.48$ & R4EI, R4EII, R4EIV, R4EV & GPBD 4, TAG 24 \\
Pod length (PLN) (mm) & - & - & - & - \\
Pod width (PWD) (mm) & - & - & - & - \\
Seed length (SLN) (mm) & 2 & $4.30-5.34$ & R4EII & GPBD 4, TAG 24 \\
Yield per plant (YPP) (gm) & 9 & $5.06-15.06$ & R4EII, R4EIV, R4EV, R4EVII & GPBD 4, TAG 24 \\
Yield Kg per hectare (YKGH) (kg) & 3 & $4.17-8.20$ & R4EII, R4EIII & GPBD 4 \\
Hundred seed weight (HSW) (gm) & 8 & $5.68-9.14$ & R4EI, R4EIII, R4EIV, R4EV, R4EVII & TAG 24, GPBD 4 \\
Shelling percentage (SLNG) (\%) & 7 & $5.03-14.04$ & R4EI, R4EIV & GPBD 4, TAG 24 \\
Days to maturity (DM) (days) & - & & &
\end{tabular}

clusters were identified where QTLs were detected for more than three traits in the linkage groups AhV, AhVI, $\mathrm{AhXV}$, and AhXVIII. For instance, a 4.9-cM region (GM633-PM179) on AhV mapped at position 70.41$70.51 \mathrm{cM}$ carried four co-localized M-QTLs contributing for PLHT, YKGH, HSW, and SLNG, and was detected in six environments (R4EI, R4EII, R4EIII,
R4EIV, R4EV, and R4EVI) explaining 5.97-9.67\% PVE. Similarly, the M-QTL with flanking markers TC1A01-S108 in the LG AhVI (7.61-12.71 cM) was found to be clustered for traits LLN, SLN, and HSW in R4EII, R4EIII, R4EIV, and R4EV and it explained 5.348.65\% PVE. Another co-mapped M-QTL (GM1536GM2301) for PPP, YKGH, and HSW detected on
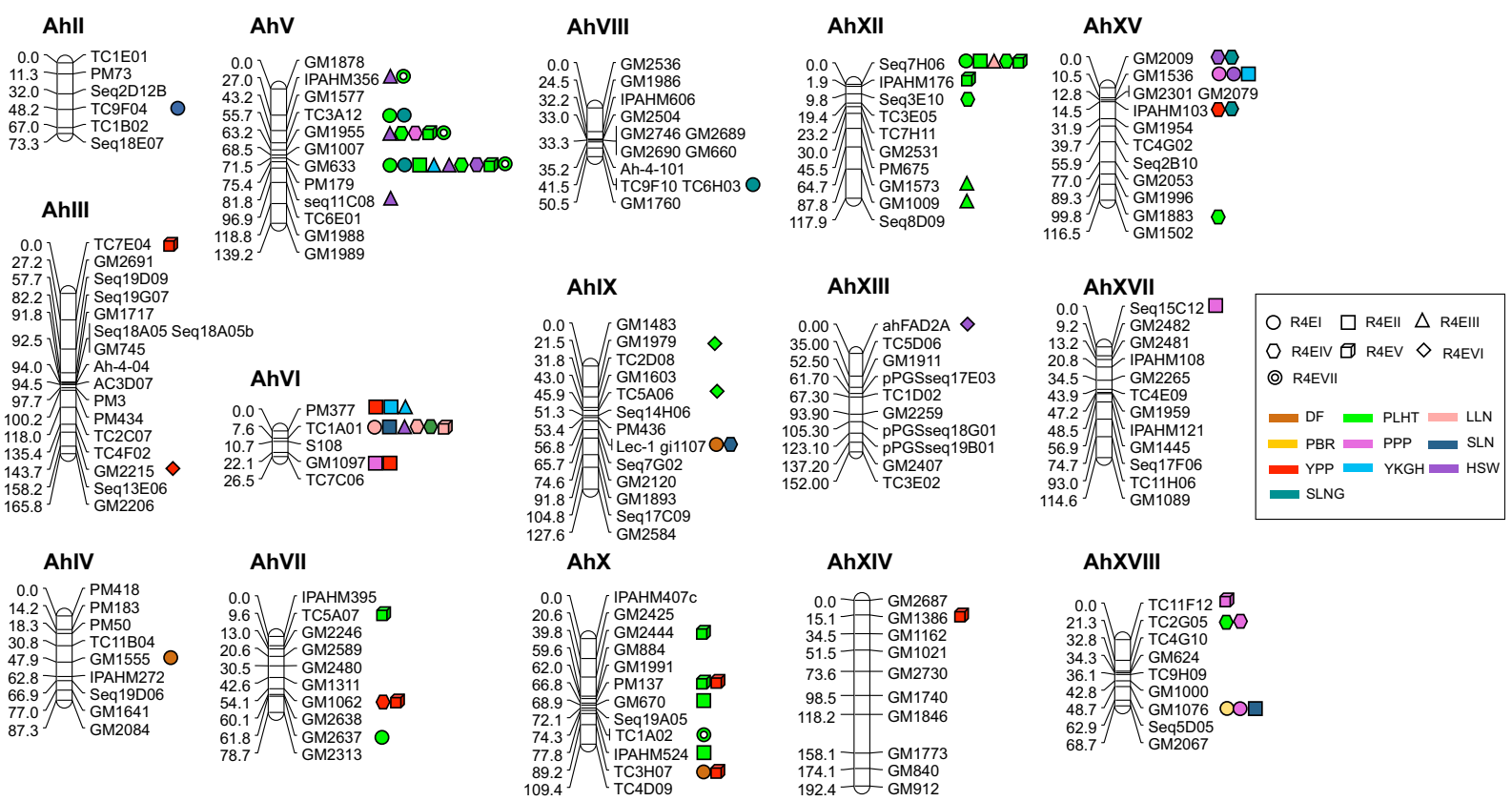

Fig. 2 Genetic linkage map showing the location of main effect QTLs identified using composite interval mapping (CIM) for agromorphological traits among the RILs of peanut 
AhXV at 10.51-12.51 cM appeared in two (R4EI and R4EII) of the seven environments, which explained 4.17-8.82\% PVE. One of the major M-QTL (IPAHM103-Seq19D6/IPAHM103-GM1954) was mapped on AhXV (14.51-20.51 cM), responsible for rust resistance exhibited pleiotropic effects for two yield-contributing traits such as YPP and SLNG explaining 15.06 and $13.40 \%$ PVE, respectively. A MQTL on AhXVIII flanked by markers GM1076Seq5D05 mapped at position 58.71-62.91 cM was colocalized for the traits like PLHT (R4EIII), PBR (R4EI), PPP (R4EI), and SLN (R4EII) with 4.30-10.48\% PVE (Supplementary Table S2). Apart from these clusters, M-QTLs for PLHT, PPP, and HSW were tagged with markers GM1955-GM1007 on LG AhV (63.21$65.21 \mathrm{cM}$ ) and were detected in four (R4EIII, R4EIV, R4EV, and R4EVI) of the seven environments that accounted for 5.11-8.06\% PVE. A co-localized MQTL for PLHT and SLNG in the marker interval of TC3A12-GM1955 on LG AhV was detected only in R4EI and accounted for $5.52-11.57 \%$ PVE. This MQTL was detected at high LOD (6.37) and had a major effect on PLHT (11.57\% PVE). The M-QTL flanked by GM2009-GM1536 mapped on AhXV (2.01-10.01 cM) co-segregated with HSW and SLNG accounted for 6.17 and $14.04 \%$ PVE, respectively.

Of the 16 co-mapped M-QTLs, eight minor effect MQTLs co-segregated with a combination of two traits with PVE ranged from 3.84-9.73\%. These QTL regions included PM137-GM670 (PLHT and YPP), IPAHM356-GM1577 (PLHT and HSW), PM377TC1A01 (YPP and YKGH), Seq7H06-IPAHM176 (PLHT and LLN), TC2G05-TC4G10 (PLHT and PPP), TC3H07-TC4D09 (DF and YPP), GM1097TC7C06 (PPP and YPP), and gi1107-Seq7G02 (DF and SLN).

\section{Epistatic QTLs}

An effort was made to check the M-QTLs that interacted with each other and to measure the nature and the extent of interaction. In total, 24 E-QTLs were mapped for all traits except LWD, PBR, and PPP (Table 2; Supplementary Table S3; Fig. 3). Of these, 16 E-QTLs showed significant additive $\times$ additive epistasis $\times$ environment (AAE) interactions $(0.01-2.81 \%$ PVE) for ten traits whereas other eight E-QTLs showed only additive $\times$ additive (AA) effects (0.03-4.49\% PVE). Among 24 E-QTLs, one E-QTL pair was mapped for DM and
PWD, two each for DF, PLN, SLN, YPP, YKGH, and SLNG, three for LLN and PLHT, and four for HSW were identified. In general, PVE by E-QTLs was very low due to epistatic interactions. The PVE for E-QTL was observed for SLN (4.28-4.49\% PVE) followed by PLN (1.21-3.34\% PVE), SLNG (1.75-3.03\% PVE), PLHT (1.71-2.91\% PVE), YKGH (1.55-2.41\% PVE), and HSW (0.36-2.41\% PVE).

Of the 16 E-QTLs, only five of them involved the MQTLs. For instance, an M-QTL at TC3A12-GM1955 on $\mathrm{AhV}$ showed an epistatic (additive $\times$ additive) interaction with the M-QTL at GM670-Seq19A05 on AhX governing PLHT with a PVE of $1.21 \%$. Such epistatic interactions were also observed for TC5A06-Seq14H06 $($ AhIX) $\times$ GM1536-GM2301 (AhXV) influencing PLHT $\times$ PPP, PLHT $\times$ YKGH, and PLHT $\times$ HSW, and TC9F04-TC1B02 (AhII) $\times$ GM1555-IPAHM272 $($ AhIV) influencing SLNG $\times$ DF. TC3A12-GM1955 $\times$ GM670-Seq19A05 and TC9F04-TC1B02 × GM1555IPAHM272 also showed QE (additive $\times$ additive $\times$ environment) interactions. It was interesting to note that the M-QTL at TC3A12-GM1955 which showed epistatic interaction was a major M-QTL.

\section{Discussion}

Yield and yield-contributing traits are quantitative in nature and show complex inheritance because their phenotypic expression is dependent on a combination of minor genes, modifiers, and environments (Kover et al. 2009). Genetic dissection of potential genomic regions harboring QTLs associated with these traits is expected to reveal the genetic control of the trait. Identification of QTLs with main effects and epistatic effects is essential for the efficient marker-assisted selection (MAS) aimed at improving breeding efficiency (Bocianowski 2013). An epistatic QTL implies that the effects of single-locus QTLs are mostly dependent on the genotypes of other loci. Thus, breeding programs have to take into account the epistatic effects while employing QTLs.

The classic example for identifying the main-effect QTLs in peanut are for resistance to rust and late leaf spot and high oleic acid which were successfully deployed in developing molecular breeding products in peanut (see Vishwakarma et al. 2017). There have been some reports of using epistatic QTLs in plant breeding to confirm the universality and the importance of epistasis between QTLs and provide useful information for 
Table 2 Epistatic QTL pairs detected for agronomic traits in RIL-4 mapping population

\begin{tabular}{llll}
\hline Trait & Number of E-QTLs & AA (PVE \%) & Environments (AAE) \\
\hline Days to flowering (DF) (days) & 2 & $1.20-1.27$ & R4EI, R4EIII \\
Plant height (PLHT) (cm) & 3 & $1.71-2.91$ & R4EIV, R4EV \\
Leaf length (LLN) (mm) & 3 & $0.03-1.12$ & R4EIV \\
Leaf width (LWD) (mm) & - & - & - \\
Primary branching (PBR) & - & - & - \\
Pods per plant (PPP) (mm) & - & - & - \\
Pod length (PLN) (mm) & 2 & $1.21-3.34$ & R4EIII \\
Pod width (PWD) (mm) & 1 & 3.34 & - \\
Seed length (SLN) (mm) & 2 & $4.28-4.49$ & R4EIII \\
Yield per plant (YPP) (gm) & 2 & $0.64-0.88$ & R4EV \\
Yield Kg per hectare (YKGH) (kg) & 2 & $1.55-2.41$ & R4EIII \\
Hundred seed weight (HSW) (gm) & 4 & $0.36-2.02$ & R4EIV \\
Shelling percentage (SLNG) (\%) & 2 & $1.75-3.03$ & R4EIV \\
Days to maturity (DM) (days) & 1 & 1.81 & R4EI
\end{tabular}

$A A$ additive $\times$ additive interactions, $A A E$ additive $\times$ additive epistasis $\times$ environment

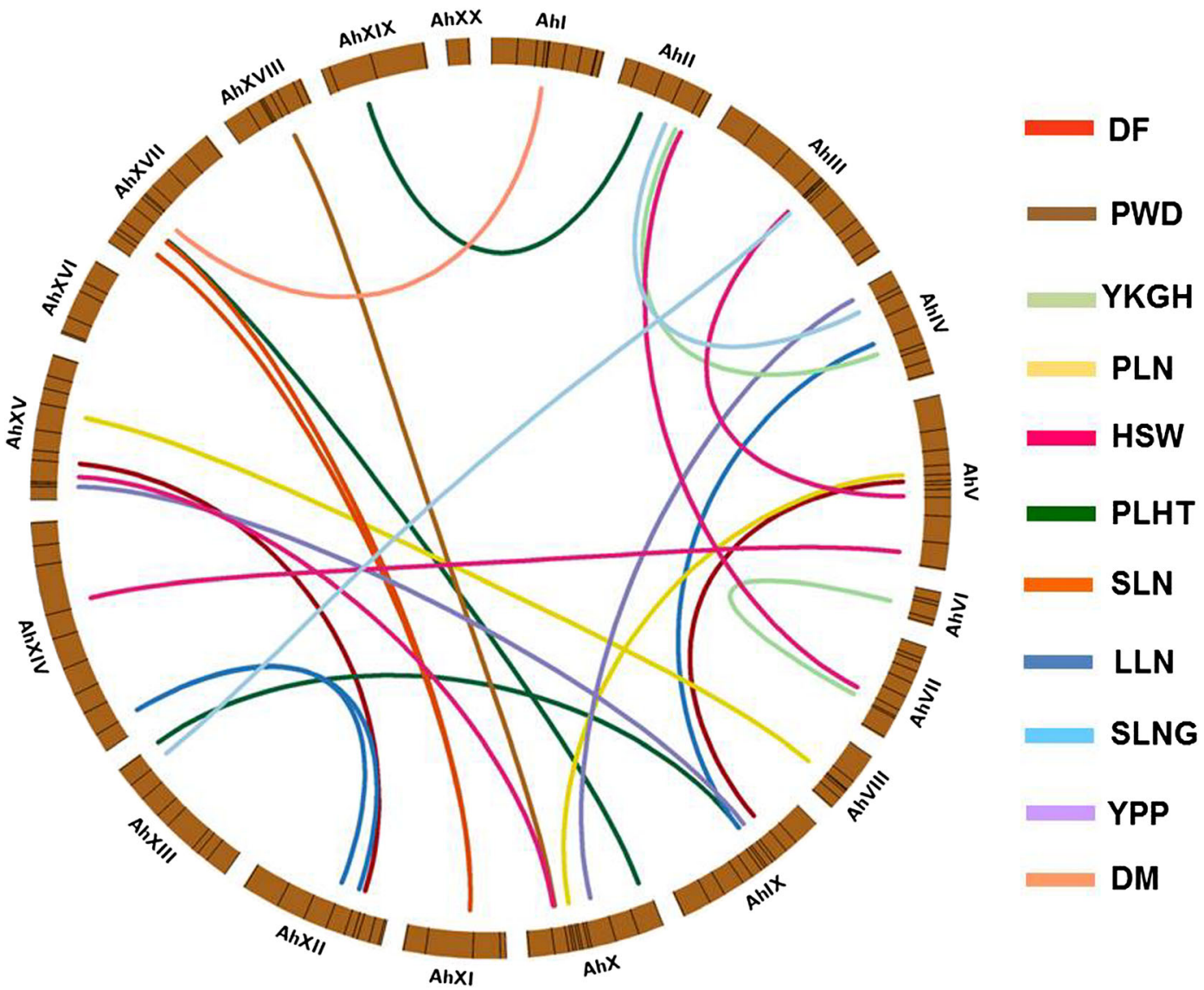

Fig. 3 Epistatic (E-QTLs) QTLs identified for agro-morphological traits among the RILs of peanut. In this figure, the brown circumference of the circus are linkage groups (LGs) with marker positions depicted as black cross lines within LGs. The interacting QTL pairs are represented with network lines inside the circus plot 
improving plant height via heterosis and QTL pyramiding in rice (Zhu et al. 2015).

Most of the QTL analysis conducted for agronomic traits in peanut till date reported just M-QTLs and therefore provided no idea on environment interactions (Kolekar et al. 2016; Hake et al. 2017; Luo et al. 2017a; Luo et al. 2017b). However, few studies provided information on both type of QTLs (M-QTLs and E-QTLs) for traits such as drought-related traits (Ravi et al. 2011; Gautami et al. 2012), fatty acids (Pandey et al. 2014b; Wang et al. 2015), and pod- and kernel-related traits (Chen et al. 2016a). The detailed information on main effect and environment interactions for the loci associated with traits provide better understanding on their contribution towards trait development, which helps in devising strategy for improving the studied trait through different available GAB approaches.

In this study, M-QTLs and E-QTLs were identified for the important agro-morphological and productivity traits from a RIL mapping population which was phenotyped for multi-seasons at multiple environments. In general, phenotypic and genotypic variability and heritability estimates were high for majority of the traits indicating to low $\mathrm{G} \times \mathrm{E}$ interactions. Nevertheless, low heritability (for YPP in R4EIV and HSW in R4EII) indicated low genetic variability due to the influence of environment on these traits. Strong positive correlations between most of the yield-related traits indicated the possibility of common genes/pathways operating at molecular level, thus sharing common genomic regions whereas negative correlation among PLHT with PLN, SLN, and SLNG; PPP with PLN, PWD, and SLN; SLNG with DF, PLHT, SLN, PWD, PLN, and PBR indicates the possibility of independent inheritance of these traits. In addition, the strong positive correlations among traits can help in simultaneous improvement of traits through modern breeding approaches.

The RIL population with high variability and normal distribution of means was considered for QTL analysis using a genetic map reported by our group (Sujay et al. 2012). One or more M-QTLs were detected for ten traits, while no M-QTLs could be detected for four traits (LWD, PLN, PWD, and DM) probably due to nonsignificant differences among the RILs and skewed distribution of means. Five M-QTLs governed two traits each, and these traits (PPP, SLN, YPP, YKGH, and HSW) were significantly and positively correlated in many environments. Of these $62 \mathrm{M}-\mathrm{QTLs}$, only six were major (PVE > 10\%) and they governed YPP
(13.56 and $15.06 \%)$, SLNG (13.40 and 14.04\%), PLHT (11.57\%), and PPP (10.48\%). These M-QTLs were detected with at least 5.0 LOD. GPBD 4 contributed the favorable alleles at all the major M-QTLs, indicating a possibility of using this parent as donor in the breeding programs.

Stability of the M-QTL is an important parameter deciding the utility of QTLs. Though none of the major M-QTLs were stable across the environments, a few with relatively high PVE were stable. For PLHT, a MQTL (71.51-73.51 cM) on AhV with a maximum PVE and LOD of 9.67 and $7.09 \%$, respectively was found stable across five environments. Another M-QTL on AhXII for PLHT with a maximum PVE and LOD of 7.12 and $5.03 \%$, respectively appeared across four environments. Likewise, stable M-QTLs were observed for LLN (7.61-12.71 cM, TC1A01-S108, AhVI), YPP (56.11-60.11 cM, GM1062-GM2638, AhVII), YKGH (0.01 cM, PM377-TC1A01, AhVI), and HSW (70.5171.51 cM, GM633-PM179, AhV).

Pleiotropic M-QTLs (QTLs governing multiple traits) and co-localized M-QTLs (QTLs located in adjacent genomic regions) are important for the simultaneous improvement of multiple traits. In this study, five pleiotropic M-QTLs governing PPP + HSW, YPP + YKGH, SLN + HSW, PPP + YPP, and PPP + YKGH were identified. At sixteen genomic regions, the $\mathrm{M}$ QTLs governing two to four productivity traits were co-localized. Marker-assisted breeding for these traits could be favored due to pleiotropy and co-localization of the M-QTLs. Such co-localized QTLs were also observed in the previous studies (Ravi et al. 2011; Gautami et al. 2012; Sujay et al. 2012; Pandey et al. 2014b; Wang et al. 2015) for traits like drought, foliar diseases, and fatty acid contents.

A previously identified marker linked to rust resistance (Khedikar et al. 2010; Sujay et al. 2012) was found to be associated with YPP (15.60\% PVE) and SLNG (13.40\% PVE) in R4EIV in this study. The introgression of this genomic region in the elite peanut varieties has not only enhanced rust resistance but increased the yield by $30-70 \%$ (Varshney et al. 2014; Yeri and Bhat 2016; Kolekar et al. 2017). This genomic region was dissected to identify genes (Pandey et al. 2017b), and the transcriptomic analysis showed differential expression of these genes among the genotypes differing for LLS resistance (Han et al. 2017).

Inter-allelic interactions among two QTLs/genes lead to E-QTLs, which play a key role in controlling trait 
expression, and are considered major components controlling qualitative and quantitative traits ( $\mathrm{Yu}$ et al. 1997). In the present study, 24 E-QTLs were identified by two-locus analysis accounting for small effects $(<$ $5 \%)$ compared to single locus main effect QTLs. This showed that E-QTLs associated with agromorphological traits encompass QTL interactions QTL $\times$ environment $(\mathrm{QE})$ interactions. QTL with more than one digenic interactions was observed in the present study; of which, 16 also showed QE interactions. Five epistatic QTLs involved the M-QTLs identified in this study, and they influenced PLHT $\times$ PLHT, PLHT $\times$ PPP, $\mathrm{PLHT} \times \mathrm{YKGH}, \mathrm{PLHT} \times \mathrm{HSW}$, and SLNG $\times \mathrm{DF}$. However, in all these cases, the PVE was relatively low (0.64-15.5\% PVE), and the major M-QTLs were not involved in any epistatic interactions, indicating that they can be considered for marker-assisted breeding without considering the need for transferring their interacting QTLs, which could simplify the breeding program.

\section{Conclusions}

The present study identified several major and minor effect M-QTLs for various economically important agro-morphological and productivity traits. Six major QTLs were detected for yield-attributing traits that can be validated using either mapping populations with different genetic background or germplasm lines and later can be utilized in peanut improvement programs using marker-assisted selection (MAS). In the present study, the favorable alleles at most of the M-QTLs and E-QTLs were contributed by GPBD 4, thereby easing the process of their transfer to the elite genotypes. The epistatic effects were not very high for the small effect MQTLs and major effect M-QTLs, which again would suggest a relatively simple backcross breeding scheme. With the current next generation sequencing (NGS) and next generation mapping (NGM), the genomic regions contributing significantly for the productivity traits can be dissected for the candidate gene(s).

Acknowledgements This work has been undertaken as part of the CGIAR Research Program on Grain Legumes. ICRISAT is a member of CGIAR Consortium. The authors would like to thank Erin Higgins for valuable comments to improve the quality of the manuscript.
Funding information The work presented in this article is a contribution from research projects sponsored by National Funds for Basic Strategic and Frontier Application Research in Agriculture (NFBSFARA) of Indian Council of Agricultural Research (ICAR), New Delhi, India, and World Bank assisted Karnataka Watershed Development Project-II (KWDP-II) funded by Government of Karnataka (GoK), India.

\section{Compliance with ethical standards}

Competing interests The authors declare that they have no competing interests.

\section{References}

Bertioli DJ, Cannon SB, Froenicke L, Huang G, Farmer AD, Cannon EK, Liu X, Gao D, Clevenger J, Dash S, Ren L, Moretzsohn MC, Shirasawa K, Huang W, Vidigal B, Abernathy B, Chu Y, Niederhuth CE, Umale P, Araujo AC, Kozik A, Do Kim K, Burow MD, Varshney RK, Wang X, Zhang X, Barkley N, Guimaraes PM, Isobe S, Guo B, Liao B, Stalker HT, Schmitz RJ, Scheffler BE, Leal-Bertioli SC, Xun X, Jackson SA, Michelmore R, Ozias-Akins P (2016) The genome sequences of Arachis duranensis and Arachis ipaensis, the diploid ancestors of cultivated peanut. Nat Genet 48(4):438-446. https://doi.org/10.1038/ng.3517

Bocianowski J (2013) Epistasis interaction of QTL effects as a genetic parameter influencing estimation of the genetic additive effect. Genet Mol Biol 36(1):093-100. https://doi. org/10.1590/S1415-47572013000100013

Carlborg Ö, Haley CS (2004) Epistasis: too often neglected in complex trait studies? Nat Rev Genet 5(8):618-625. https://doi.org/10.1038/nrg1407

Chen W, Jiao Y, Cheng L, Huang L, Liao B, Tang M, Ren X, Zhou X, Chen Y, Jiang H (2016a) Quantitative trait locus analysis for pod-and kernel-related traits in the cultivated peanut (Arachis hypogaea L.) BMC Genet 17(1):25. https://doi. org/10.1186/s12863-016-0337-x

Chen X, Li H, Pandey MK, Yang Q, Wang X, Garg V, Chi X, Doddamani D, Hong Y, Upadhyaya H, Guo H, Khan AW, Zhu F, Zhang X, Pan L, Pierce GJ, Zhou G, Krishnamohan KA, Chen M, Zhong N, Agarwal G, Li S, Chitikineni A, Zhang GQ, Sharma S, Chen N, Liu H, Janila P, Wang M, Wang T, Sun J, Li X, Li C, Yu L, Wen S, Singh S, Yang Z, Zhao J, Zhang C, Yu Y, Bi J, Liu ZJ, Paterson AH, Wang S, Liang X, Varshney RK, Yu S (2016b) Draft genome of the peanut A-genome progenitor (Arachis duranensis) provides insights into geocarpy, oil biosynthesis, and allergens. Proc Natl Acad Sci 113(24):6785-6790. https://doi.org/10.1073 /pnas. 1600899113

Dwivedi S, Thendapani K, Nigam S (1989) Heterosis and combining ability studies and relationship among fruit and seed characters in peanut. Peanut Sci 16(1):14-20. https://doi. org/10.3146/i0095-3679-16-1-4

FAOSTAT (2016) FAO statistical database. http://faostat.fao.org 
Gautami B, Pandey MK, Vadez V, Nigam SN, Ratnakumar P, Krishnamurthy L, Radhakrishnan T, Gowda MV, Narasu ML, Hoisington DA, Knapp SJ, Varshney RK (2012) Quantitative trait locus analysis and construction of consensus genetic map for drought tolerance traits based on three recombinant inbred line populations in cultivated groundnut (Arachis hypogaea L.) Mol Breed 30(2):757-772. https://doi. org/10.1007/s11032-011-9660-0

GenStat Committee (2010) Genstat v12.2, VSN International. http://www.vsni.co.uk/downloads/genstat/12th-editionupgrade

Gowda MVC, Motagi BN, Naidu GK, Diddimani SB, Sheshagiri R (2002) GPBD 4: a spanish bunch groundnut genotype resistant to rust and late leaf spot. Int Arachis News Lett 22: 29-32

Hake AA, Shirasawa K, Yadawad A, Sukruth M, Patil M, Nayak SN, Lingaraju S, Patil PV, Nadaf HL, Gowda MVC, Bhat RS (2017) Mapping of important taxonomic and productivity traits using genic and non-genic transposable element markers in peanut (Arachis hypogaea L.) PLoS One 12(10):e0186113. https://doi.org/10.1371/journal. pone.0186113 eCollection 2017

Han S, Liu H, Yan M, Qi F, Wang Y, Sun Z, Huang B, Dong W, Tang F, Zhang X (2017) Differential gene expression in leaf tissues between mutant and wild-type genotypes response to late leaf spot in peanut (Arachis hypogaea L.) PLoS One 12(8):e0183428. https://doi.org/10.1371/journal. pone. 0183428

Khedikar Y, Gowda MVC, Sarvamangala C, Patgar K, Upadhyaya HD, Varshney R (2010) A QTL study on late leaf spot and rust revealed one major QTL for molecular breeding for rust resistance in groundnut (Arachis hypogaea L.) Theor Appl Genet 121(5):971-984. https://doi.org/10.1007/s00122-0101366-X

Kolekar RM, Sujay V, Shirasawa K, Sukruth M, Khedikar YP, Gowda MVC, Pandey MK, Varshney RK, Bhat RS (2016) QTL mapping for late leaf spot and rust resistance using an improved genetic map and extensive phenotypic data on a recombinant inbred line population in peanut (Arachis hypogaea L.) Euphytica 209(1):147-156. https://doi. org/10.1007/s10681-016-1651-0

Kolekar RM, Sukruth M, Nadaf HL, Motagi BN, Lingaraju S, Patil PV, Bhat RS (2017) Marker-assisted backcrossing to develop foliar disease resistant genotypes in TMV 2 variety of peanut (Arachis hypogaea L.). Plant Breed. https://doi. org/10.1111/pbr.12549

Kover PX, Valdar W, Trakalo J, Scarcelli N, Ehrenreich IM, Purugganan MD, Durrant C, Mott R (2009) A multiparent advanced generation inter-cross to fine-map quantitative traits in Arabidopsis thaliana. PLoS Genet 5(7):e1000551. https://doi.org/10.1371/journal.pgen.1000551

Layrisse A, Wynne J, Isleib T (1980) Combining ability for yield, protein and oil of peanut lines from South American centers of diversity. Euphytica 29(3):561-570. https://doi. org/10.1007/BF00023203

Luo H, Ren X, Li Z, Xu Z, Li X, Huang L, Zhou X, Chen Y, Chen W, Lei Y, Liao B, Pandey MK, Varshney RK, Guo B, Jiang X, Liu F, Jiang H (2017a) Co-localization of major quantitative trait loci for pod size and weight to a $3.7 \mathrm{cM}$ interval on chromosome A05 in cultivated peanut (Arachis hypogaea L.)
BMC Genomics 18(1):58. https://doi.org/10.1186/s12864016-3456-x

Luo H, Xu Z, Li Z, Li X, Lv J, Ren X, Huang L, Zhou X, Chen Y, Yu J (2017b) Development of SSR markers and identification of major quantitative trait loci controlling shelling percentage in cultivated peanut (Arachis hypogaea L.). Theor Appl Genet 1-14

Pandey MK, Upadhyaya HD, Rathore A, Vadez V, Sheshshaye MS, Sriswathi M, Govil M, Kumar A, Gowda MVC, Shivali S, Hamidou F, Anil Kumar V, Khera P, Bhat RS, Khan Amir W, Sube S, Hongjie L, Emmanuel M, Nadaf HL, Mukri G, Liang X, Jackson S, Varshney RK (2014a) Genomewide association studies for 50 agronomic traits in peanut using the reference set comprising 300 genotypes from 48 countries of semi-arid tropics of the world. PLoS One 9(8):e105228. https://doi.org/10.1371/journal.pone.0105228

Pandey MK, Wang ML, Qiao L, Feng S, Khera P, Wang H, Tonnis B, Barkley NA, Wang J, Holbrook CC (2014b) Identification of QTLs associated with oil content and mapping FAD2 genes and their relative contribution to oil quality in peanut (Arachis hypogaea L.) BMC Genet 15(1):133. https://doi. org/10.1186/s12863-014-0133-4

Pandey MK, Agarwal G, Kale SM, Clevenger J, Nayak SN, Sriswathi M, Chitikineni A, Chavarro C, Chen X, Upadhyaya HD (2017a) Development and evaluation of a high density genotyping 'Axiom_Arachis' array with $58 \mathrm{~K}$ SNPs for accelerating genetics and breeding in groundnut. Sci Rep 7:40577. https://doi.org/10.1038/srep40577

Pandey MK, Khan AW, Singh VK, Vishwakarma MK, Shasidhar Y, Kumar V, Garg V, Bhat RS, Chitikineni A, Janila P, Guo B, Varshney RK (2017b) QTL-seq approach identified genomic regions and diagnostic markers for rust and late leaf spot resistance in groundnut (Arachis hypogaea L.) Plant Biotechnol J 15(8):927-941. https://doi.org/10.1111 /pbi.12686

Patil S, Kale D, Deshmukh S, Fulzele G, Weginwar B (1995) Semi-dwarf, early maturing and high yielding new groundnut variety, TAG-24. J Oilseeds Res 12:254-257

R Core Team (2013) R: A language and environment for statistical computing. R Foundation for Statistical Computing, Vienna

Ravi K, Vadez V, Isobe S, Mir RR, Guo Y, Nigam SN, Gowda MVC, Radhakrishnan T, Bertioli DJ, Knapp SJ (2011) Identification of several small main-effect QTLs and a large number of epistatic QTLs for drought tolerance related traits in groundnut (Arachis hypogaea L.) Theor Appl Genet 122(6):1119-1132. https://doi.org/10.1007/s00122-010$1517-0$

Sujay V, Gowda MVC, Pandey MK, Bhat RS, Khedikar YP, Nadaf HL, Gautami B, Sarvamangala C, Lingaraju S, Radhakrishan T, Knapp SJ, Varshney RK (2012) QTL analysis and construction of consensus genetic map for foliar disease resistance based on two RIL populations in cultivated groundnut (Arachis hypogaea L.) Mol Breed 30(2):773-788. https://doi.org/10.1007/s11032-011-9661-z

Sukruth M, Paratwagh SA, Sujay V, Varshakumari, Gowda MVC, Nadaf HL, Motagi BN, Lingaraju S, Pandey MK, Varshney RK, Bhat RS (2015) Validation of markers linked to late leaf spot and rust resistance, and selection of superior genotypes among diverse recombinant inbred lines and backcross lines in peanut (Arachis hypogaea L.) Euphytica 204(2):343-351. https://doi.org/10.1007/s10681-014-1339-2 
Upadhyaya HD, Nigam S (1998) Epistasis for vegetative and reproductive traits in peanut. Crop Sci 38(1):44-49. https://doi.org/10.2135/cropsci 1998.0011183 X003800010008x

Varshney RK, Mohan SM, Gaur PM, Gangarao N, Pandey MK, Bohra A, Sawargaonkar SL, Gorantla A, Kimurto PK, Janila P (2013) Achievements and prospects of genomics-assisted breeding in three legume crops of the semi-arid tropics. Biotechnol Adv. https://doi.org/10.1016/j. biotechadv.2013.1001.1001

Varshney RK, Pandey MK, Pasupuleti J, Nigam SN, Sudini H, Gowda MVC, Sriswathi M, Radhakrishan T, Manohar SS, Patne N (2014) Marker-assisted introgression of a QTL region to improve rust resistance in three elite and popular varieties of peanut (Arachis hypogaea L.) Theor Appl Genet 127(8):1771-1781. https://doi.org/10.1007/s00122014-2338-3

Vishwakarma MK, Nayak L SN, Guo B, Wan L, Liao B, Varshney RK, Pandey MK (2017) Classical and molecular approaches for mapping of genes and quantitative trait loci in peanut (Arachis hypogaea L.). In: Varshney RK, Pandey MK, Puppala N (eds) The peanut genome. pp 93-116. https://doi. org/10.1007/978-3-319-63935-2

Wang S, Basten C, Zeng Z (2007) Windows QTL cartographer 2.5. North Carolina State University, Raleigh

Wang ML, Khera P, Pandey MK, Wang H, Qiao L, Feng S, Tonnis B, Barkley NA, Pinnow D, Holbrook CC (2015) Genetic mapping of QTLs controlling fatty acids provided insights into the genetic control of fatty acid synthesis pathway in peanut (Arachis hypogaea L.) PLoS One 10(4):e0119454. https://doi.org/10.1371/journal.pone.0119454

Yang J, Hu C, Hu H, Yu R, Xia Z, Ye X, Zhu J (2008) QTLNetwork: mapping and visualizing genetic architecture of complex traits in experimental populations. Bioinformatics 24(5):721-723. https://doi.org/10.1093 /bioinformatics/btm494
Yeri SB, Bhat RS (2016) Development of late leaf spot and rust resistant backcross lines in JL 24 variety of groundnut (Arachis hypogaea L.) Electron J Plant Breed 7(1):37-41. https://doi.org/10.5958/0975-928X.2016.00005.3

Yeri SB, Shirasawa K, Pandey MK, Gowda MVC, Sujay V, Shriswathi M, Nadaf HL, Motagi BN, Lingaraju S, Bhat ARS, Varshney RK, Krishnaraj PU, Bhat RS (2014) Development of NILs from heterogeneous inbred families for validating the rust resistance QTLs in peanut (Arachis hypogaea L.) Plant Breed 133(1):80-85. https://doi. org/10.1111/pbr.12130

Yin D, Wang Y, Zhang X, Ma X, He X, Zhang J (2017) Development of chloroplast genome resources for peanut (Arachis hypogaea L.) and other species of Arachis. Sci Rep 7(1):11649. https://doi.org/10.1038/s41598-017-12026-X

Yu SB, Li JX, Xu CG, Tan YF, Gao YJ, Li XH, Zhang Q, Maroof MAS (1997) Importance of epistasis as the genetic basis of heterosis in an elite rice hybrid. Proc Natl Acad Sci 94(17): 9226-9231. https://doi.org/10.1073/pnas.94.17.9226

Zeng ZB (1994) Precision mapping of quantitative trait loci. Genetics 136(4):1457-1468

Zhang X, Zhang J, He X, Wang Y, Ma X, Yin D (2017) Genomewide association study of major agronomic traits related to domestication in peanut. Front Plant Sci 8:1611. https://doi. org/10.3389/fpls.2017.01611

Zhao C, Qiu J, Agarwal G, Wang J, Ren X, Xia H, Guo B, Ma C, Wan S, Bertioli DJ (2017) Genome-wide discovery of microsatellite markers from diploid progenitor species, Arachis duranensis and A. ipaensis, and their application in cultivated peanut (A. hypogaea). Front Plant Sci 8. https://doi. org/10.3389/fpls.2017.01209

Zhu H, Liu Z, Fu X, Dai Z, Wang S, Zhang G, Zeng R, Liu G (2015) Detection and characterization of epistasis between QTLs on plant height in rice using single segment substitution lines. Breed Sci 65(3):192-200. https://doi.org/10.1270 /jsbbs.65.192 